\title{
Radiotherapy as a local treatment option for heterotopic ossifications in patients with spinal cord injury
}

\author{
ML Sautter-Bihl*,1, E Liebermeister ${ }^{1}$ and A Nanassy ${ }^{2}$ \\ ${ }^{1}$ Department of Radiooncology, Städtisches Klinikum Karlsruhe, Karlsruhe, Germany; ${ }^{2}$ Department of Orthopedics, \\ Klinikum Karlsbad-Langensteinbach, Germany
}

\begin{abstract}
Objective: Heterotopic ossifications (HO) occur in about $20 \%$ of patients with spinal cord injury and may seriously compromise the rehabilitation process. The aim of the present study was to ascertain whether radiotherapy (RT) administered early in the course of the disease prevents the manifestation of HO and whether in patients, whose HO have been resected, recurrence can be avoided.

Methods: Thirty-six patients (46 joints) were irradiated; 33 patients were evaluable. Mean follow up was 23.6 months. In 27 patients RT was performed as primary treatment in the inflammatory phase of soft tissue swelling that precedes manifest HO. Eleven patients were treated after resection of manifest HO, while two had primary and postoperative RT in different joints. The dose was $10 \mathrm{~Gy}$ in fractions of $2-2.5 \mathrm{~Gy}$ in 25 patients. Four patients were treated with total doses between 7.5 and $20 \mathrm{~Gy}$. Seven patients received single dose irradiation with $8 \mathrm{~Gy}$. RT was performed with $8 \mathrm{MV}$ photons after simulation on the basis of CT scans.

Results: The mean duration of follow up was 23.6 months. Thirty of the 36 irradiated patients showed no progression of HO. In three patients reossifications after therapy caused a moderate decrease in joint mobility. No relevant adverse effects were observed.

Conclusion: These results suggest that RT is a safe and effective local treatment option for spinal cord injury patients with $\mathrm{HO}$.
\end{abstract}

Spinal Cord (2000) 38, 33-36

Keywords: spinal cord injury; heterotopic ossification; radiotherapy

\section{Introduction}

Since the beginning of the century, when Dejerine et al ${ }^{1}$ reported on 'paraosteoarthropathies' in patients with spinal cord injuries (SCI), heterotopic ossification (HO) in these patients has been known as a clinically relevant problem occurring in about $20 \%$ of these patients. ${ }^{2-5}$

As HO in spinal cord injury patients may decrease the range of motion of the affected joints and thus seriously compromise rehabilitation, ${ }^{6}$ it seems desirable to find ways of either preventing or stabilising this pathological process at an early stage. Hitherto, no local therapeutic options have been established for $\mathrm{HO}$ except extensive resection. Surgery, however, should not be performed earlier than $1-1.5$ years after onset of the HO. ${ }^{4}$

As radiotherapy (RT) is well known to prevent HO when administered after total hip arthroplasty, it may be hypothesised to be an appropriate treatment option for SCI patients with $\mathrm{HO}$ as well. The purpose of the present paper is to evaluate the efficacy of RT to stop

*Correspondence: ML Sautter-Bihl, Department of Radiooncology, Klinik für Strahlentherapie, Stadtisches Klinikum Karlsruhe gGmbH, Moltkestrasse 90, 76133 Karlsruhe, Germany progression of manifest or incipient heterotopic bone formation or to prevent reossification after surgical treatment.

\section{Patients and methods}

The total number of SCI patients included in the study was 36 (four females, 32 males); the mean age was 33.6 years $(17-59$ years). Forty-six joints were irradiated (43 hips, one shoulder, one elbow, one knee). Thirtythree patients were evaluable; three were lost to followup. The mean duration of follow up was 23.6 months (4-98 months).

Diagnosis was based on clinical examination, X-ray studies $(n=10)$ and computed tomography (CT) $(n=23)$. Reevaluation in the follow up included clinical examination analysing the range of motion of the affected joint and radiography in order to assess ossifications around the hip according to the Brooker score ${ }^{7}$ or in more distal soft tissues and muscles.

In 27 patients (33 joints) RT was a primary treatment in the status of myositis. These patients had shown the respective clinical signs such as soft 
tissue swelling, hyperthermia and erythema. CT was then performed to visualize the affected region and confirm the clinical diagnosis. RT was started as soon as possible, when ossifications were minimal or even not yet visible at all. These patients were judged as having 'incipient' HO. In CT the inflammatory process was visualized as hypodensity and soft tissue swelling. Additionally, small spots of ossifications were sometimes present. Eleven patients (13 joints) had manifest ossifications which had to be resected. Postoperative RT was performed $24-36 \mathrm{~h}$ after surgery. Two of the 36 patients had primary and postoperative RT in different joints.

The majority of patients $(25 / 36)$ received $10 \mathrm{~Gy}$ in fractions of $2-2.5 \mathrm{~Gy}$. Only three patients were treated with higher doses: the first patient of all received $20 \mathrm{~Gy}$, one patient was treated with $15 \mathrm{~Gy}$ and one with $12 \mathrm{~Gy}$. One patient received only $7.5 \mathrm{~Gy}$ due to acute fever. In the second period of the study the treatment regimen was changed and the seven patients treated since 12/96 received single dose irradiation with $8 \mathrm{~Gy}$.

Radiotherapy was performed with a linear accelerator using $8 \mathrm{MV}$ photons. The treatment volume enclosed the affected region with a small safety margin. Field shape was based mainly on the findings of CT depicted as soft tissue swelling. Critical organsespecially the gonads in young patients when the hips were irradiated-were spared as far as possible by using individual blocks or special gonadal shielding. Gonadal dose was measured using a special dosimeter (Diamond Detector, PTW).

\section{Results}

\section{Treatment efficacy}

Thirty of the 33 evaluable patients showed neither progression of incipient $\mathrm{HO}$ nor recurrence of resected bone formations. In all these patients mobilisation and rehabilitation could be carried out as desired.

Analysing the treated joints, 16 of the 32 primarily irradiated hips did not show any radiographic abnormalities in the follow up. According to the grading proposed by Brooker ${ }^{7}$ two hips were scored as grade 1, six hip joints as grade 2 and eight as grade 3 (Table 1). Thus, three hips showed progression after RT.

In the postoperative group 11 hip joints were irradiated after resection of manifest HO. The two

Table 1 Radiographic abnormalities in the primary RT group (32 hip joints)

\begin{tabular}{lcccc}
\hline & None & Brooker 1 & Brooker 2 & Brooker 3 \\
\hline Before RT & 17 & 3 & 7 & 5 \\
After RT & 16 & 2 & 6 & 8 \\
\hline
\end{tabular}

Radiographic abnormalities according to Brooker grade 4 did not occur hips without radiographic abnormalities after resection remained free of any morphologic alterations in the follow up. Prior to RT four hips showed Brooker grade 1, five hips had grade 2 alterations. In the follow up three hips had Brooker grade 1, five hips grade 2 and one hip grade 3 ossifications (Table 2). Abnormalities according to Brooker grade 4 did not occur.

Radiograms of the other three irradiated joints (one knee, one elbow, one shoulder) were assessed according to the classification proposed by Garland. ${ }^{8}$ Mild radiographic abnormalities occurred in the knee, moderate alterations in the elbow and shoulder. All these findings remained unchanged after RT.

\section{Adverse effects}

No relevant adverse effects occurred. One patient treated postoperatively developed fever the day after radiotherapy probably not related to RT. Delayed wound healing in operated patients was not observed.

\section{Gonadal doses}

When the hip was irradiated, the mean gonadal dose was measured as $1.2-1.5 \mathrm{cGy}$ per Gy of total dose using gonadal shielding.

\section{Discussion}

As $\mathrm{HO}$ occurs in up to $20 \%$ of patients with spinal cord injuries and then may pose serious problems for rehabilitation, it seems desirable to establish methods for early diagnosis and treatment in order to prevent progression. $\mathrm{HO}$ is preceded by an acute inflammatory process affecting soft tissues and muscles in the neighbourhood of joints. In most cases the onset of the disease occurs between 4 weeks and 3 months (at the latest 6 months) after the initial trauma., Sometimes there is a coincidence with the phase of mobilisation and passive movements of the joints. ${ }^{10}$

Radiotherapy has not been established as a treatment for $\mathrm{HO}$ in patients with spinal cord injuries. A clinical rationale for irradiation as an appropriate treatment for HO in SCI patients may be derived from the fact that RT is an established means of preventing $\mathrm{HO}$ after hip replacement and has been performed since the early 1970s. The first trials started using $20 \mathrm{~Gy}$, but doses were then reduced to $10 \mathrm{~Gy},{ }^{11,12}$ which was regarded as standard treatment

Table 2 Radiographic abnormalities in the postoperative RT group (11 hip joints)

\begin{tabular}{lcccc}
\hline & None & Brooker 1 & Brooker 2 & Brooker 3 \\
\hline Before RT & 2 & 4 & 5 & 0 \\
After RT & 2 & 3 & 5 & 1
\end{tabular}

Radiographic abnormalities according to Brooker grade 4 did not occur 
until recently. Nowadays, prophylactic RT in high-risk patients with hip replacement is in many institutions performed in a single fraction of 7-8 Gy, which has been shown to be just as effective as the fractionated regimen while easier to perform. ${ }^{13-15}$

Moreover, it is well known that low-dose RT has an excellent antiphlogistic effect, which could particularly be expected in the inflammatory process affecting the soft tissues preceding the development of HO.

With regard to the clinical efficacy of this treatment modality in the prevention of heterotopic bone formation, RT seems to suggest itself for application in SCI patients as well. However, to our knowledge, no systemic investigations on the use of external RT for SCI patients have been published. Some publications exist describing postoperative RT after resection of $\mathrm{HO}$. In a recent study of the results of resection of $\mathrm{HO}$ in SCI patients, postoperative irradiation was used, and the excellent outcome of this combined modality treatment was attributed partly to radiotherapy. ${ }^{16}$ However, doses and technique of RT were not described in detail and no information about indication for RT or patient selection was given. Another clinical study reports excellent results in eight patients (five of which had SCI) whose HO of the elbow was resected and irradiated postoperatively. No recurrences occurred. ${ }^{17}$ Stover et $a l^{4}$ performed postoperative RT in two out of 21 patients but found the effects difficult to determine as other treatment modalities were also employed.

In another publication, which is based on two cases, pain relief and an increased range of motion was observed after RT in patients with established ossifications. $^{18}$

Our group reported preliminary results in $1995,{ }^{19}$ suggesting the efficacy of RT not only when performed postoperatively but particularly as a primary treatment in the early stage of the disease, ie in the inflammatory phase preceding the manifest ossifications. However, in this study the follow up time was too short to permit definitive conclusions.

Corresponding to the results of our preliminary study, ${ }^{19}$ the present findings confirmed the efficacy of RT as a primary treatment for incipient HO; RT was equally effective in the postoperative situation. In contrast to our first results the mean follow up time of 23.6 months now permits a more pertinent conclusion. Further progression is unlikely, as the highest frequency of occurrence is at $1-4$ months after the spinal lesion. ${ }^{2,20}$

Only three out of $33(<10 \%)$ patients developed moderate progression of their ossifications after RT. These patients were not treated at the optimal time point, ie the very beginning of the disease but after manifest onset of $\mathrm{HO}$.

There was no difference of efficacy in the fractionated regimen compared to the single dose treatment. In the first treatment period patients were irradiated with fractionated RT according to the 'gold standards' in prophylactic RT for prevention of HO in total hip arthroplasty. When some randomized trials. $^{14,15}$ had shown no difference in outcome between the two schedules in hip replacement patients, one single fraction with $8 \mathrm{~Gy}$ was used since 1996. With regard to organisational feasibility single dose treatment is favourable as specialized rehabilitation centres eventually are not equipped with a radiotherapy department and the transportation is arduous for the patients.

These results seem to compare favourably to the treatment with systemic diphosphonate treatment. Especially in older investigations the efficacy of etidronate seemed uncertain. ${ }^{8,21}$ Banovac described prevention of $\mathrm{HO}$ in $79 \%$ in patients with positive bone scan and negative radiogram and only in six of 13 patients $\mathrm{HO}$ could be prevented when patients had radiographically visible $\mathrm{HO}^{22}$ However, treatment efficacy with etidronate seems to be higher when used in a very early stage of the disease. ${ }^{23}$ Moreover, RT, especially when performed as single dose treatment, is extremely cost effective compared to several months of medical treatment.

No relevant adverse effects have yet been observed in our patients. However, the potential long-term risk of a radiogenic tumor induction for patients of predominantly young age has to be taken into consideration. There seems to be no relevant risk of a local tumor induction within the radiation field (ie sarcoma) as this is not supposed to occur after doses smaller than $30 \mathrm{~Gy}^{24}$ The potential actuarial risk of systemic tumor induction (years or decades after treatment) is difficult to quantify as data are derived from a single whole-body exposition especially at Hiroshima and Nagasaki, ${ }^{25,26}$ whereas in the therapeutic situation only a small part of the body is exposed to relevant doses. These small risks must be weighed carefully against the risk of hampered rehabilitation with serious impairment of life quality, particularly in the absence of other treatment options with proven efficacy.

As patients with SCI are often young males, some of whom will wish to father children, special care has to be taken to preserve fertility. This is especially important in view of the fact that modern fertilization techniques permit reproduction even in patients with a loss of potency. We therefore used careful gonadal shielding with a special lead capsule. The measured gonadal dose was only $10 \%$ to $15 \%$ of the dose (1Gy) considered critical for infertility. ${ }^{27}$

\section{Conclusion}

The results of the present study confirm that RT for the treatment of $\mathrm{HO}$ in patients with SCI is effective as a primary therapy as well as postoperatively. When used as a primary treatment RT should be performed as early as possible after the onset of the disease. A prospective study evaluating the efficacy of single dose radiotherapy is currently under way. 


\section{References}

1 Dejerine M, Ceillier A. Para-Osteo-Arthropathie des paraplegiques par lesions medullaires. Ann Med Paris 1918; 5: 497-535.

2 Garland DE. A clinical perspective on common forms of acquired heterotopic ossification. Clin Orthop 1991; 263: 13-29.

3 Hernandez AM et al. The para-articular ossifications in our paraplegics and tetraplegics: a survey of 704 patients. Paraplegia 1978; 16: $272-275$.

4 Stover SL, Niemann KMW, Tulloss JR. Experience with surgical resection of heterotopic bone in spinal cord injury patients. Clin Orthop 1991; 263: $71-77$.

5 Wittenberg RH, Peschke U, Bötel U. Heterotopic ossification after spinal cord injury. Epidemiology and risk factors. $J$ Bone Joint Surg (BR) 1992; 74: 215-218.

6 Wharton GW. Heterotopic ossification. Clin Orthop 1975; 112: $142-149$.

7 Brooker AF, Bowerman JW, Robinson RA, Riley LH. Ectopic ossification following total hip replacement. J Bone Joint Surg 1973; 55A: $1629-1632$.

8 Garland DE, Alday B, Venos KG, Vogt JC. Diphosphonate treatment for heterotopic ossification in spinal cord injury patients. Clin Orthop 1983; 176: 197-200.

9 Wharton GW, Morgan TH. Ankylosis in the paralyzed patient. J Bone Joint Surg 1970; 521: 105-112.

10 Daud O, Sett P, Burr RG, Silver JR. The relationship of heterotropic ossification to passive movements in paraplegic patients. Disability Rehabilitation 1993; 15: 114-118.

11 Anthony $\mathrm{P}$ et al. Prevention of heterotopic bone formation. Int $J$ Radiat Oncol Biol Phys 1987; 13: 365 - 369.

12 Sylvester JE et al. The use of post-operative irradiation for the prevention of heterotopic bone formation after total hip replacement. Int J Radiat Oncol Biol Phys 1988; 14: 471-476.

13 Konski et al. Randomized trial comparing single dose versus fractionated irradiation for prevention of heterotopic bone: a preliminary report. Int J Radiat Oncol Biol Phys 1990; 18: $1139-$ 1142.

14 Gregoritch SJ et al. Randomized trial comparing preoperative versus postoperative irradiation for prevention of heterotopic ossification following prosthetic total hip replacement. Preliminary results. Int J Radiat Oncol Biol Phys 1994; 30: 55-62.
15 Seegenschmiedt MH et al. Preoperative versus postoperative radiotherapy for prevention of heterotopic ossification (HO). First results of a randomized trial in high risk patients. Int $J$ Radiat Oncol Biol Phys 1994; 30: 63-73.

16 Meiners T, Abel R, Böhm V, Gerner HJ. Resection of heterotopic ossification of the hip in spinal cord injured patients. Spinal Cord 1997; 35: $443-445$.

17 McAuliffe JA, Wolfson AH. Early excision of heterotopic ossification about the elbow followed by radiotherapy. $J$ Bone Surg Am 1997; 79: 749-755.

18 Schaeffer MA, Sosner J. Heterotopic ossification: treatment of established bone with radiation therapy. Arch Phys Med Rehabil 1995; 76: $284-286$

19 Sautter-Bihl ML et al. Strahlentherapie heterotoper Ossifikationen bei Querschnittsgelähmten. Strahlenther Onkol 1995; 171: $454-459$.

20 Bravo-Payno P. Incidence and risk factors in the appearance of heterotopic ossification in spinal cord injury. Paraplegia 1992; 30: $740-745$.

21 Orzel JA, Rudd TG. Heterotopic bone formation: Clinical, laboratory and imaging correlation. J Nucl Med 1985; 26: 125 132.

22 Banovac K, Gonzalez F, Renfree KJ. Treatment of heterotopic ossification after spinal cord injury. J Spinal Cord Med 1997; 20: $60-65$.

23 Banovac K, Gonzales F. Evaluation and management of heterotopic ossification in patients with spinal cord injury. Spinal Cord 1997; 35: $158-162$

24 Brady LW. Radiation induced sarcomas of bone. Skel Radiol 1979; 4: $72-78$.

25 BEIR Committee on the Biological Effects of Ionizing Radiation. Health effects of exposure to low levels of ionizing radiation. National Academy Press, Washington DC 1990.

26 International Commission on Radiological Protection: Recommendations. Annals of the ICRP, No 60. Pergamon Press. Oxford 1990

27 Greiner R. Die Erholung der Spermatogenese nach fraktionierter, niedrigdosierter Bestrahlung der männlichen Gonaden. Strahlenther Onkol 1982; 158: 342-355. 